\title{
Cisticercosis Humana en el Ecuador
}

\section{Human Cysticercosis in Ecuador}

\author{
Vilma Amanda Uguña Rosas ${ }^{1 *}$ \\ ${ }^{1}$ Universidad Católica de Cuenca \\ *amandauquimica@hotmail.com
}

DOI: https://doi.org/10.26871/killkana_salud.v2i2.269

\begin{abstract}
Resumen
Objetivo: El presente artículo se propone analizar mediante una revisión bibliográfica de la cisticercosis humana a nivel de Latinoamérica y en el Ecuador, resaltando las características más relevantes de Taenia solium que es el parásito que produce esta enfermedad; como el ciclo de vida, la epidemiología, manifestaciones clínicas, problema socioeconómico, así como las técnicas de diagnóstico, mecanismos de infección, el tratamiento y los programas de control que se han venido ejecutando en diversos países desarrollados con el objetivo de erradicar la enfermedad. Contexto: La cisticercosis humana es una enfermedad zoonótica la cual es producida por el consumo de huevos de T. solium de manera accidental; esta puede causar trastornos graves de salud e incluso la muerte. Si bien los datos de prevalencia están disponibles en todo el mundo, la tasa de incidencia y las cifras de incidencia acumulada son insuficientes, lo que limita la comprensión de la epidemiología de T. solium, se la considera como una de las infecciones que ocurre con mayor frecuencia en el sistema nervioso; además es endémica en varios países de América Latina, Asia y África. Metodología: En este artículo se realizó una revisión bibliográfica de la información que se encuentra disponible sobre la cisticercosis y su parásito causante $T$. solium; así como los programas de control que se realizaron en los países desarrollados y que lograron erradicar esta enfermedad.
\end{abstract}

Palabras clave: Cisticercosis, Taenia solium, neurocisticercosis.

\begin{abstract}
Objective: The present article proposes to analyze through a bibliographic review of human cysticercosis at the of Latin America and in Ecuador, highlighting the most relevant characteristics of Taenia solium, which is the parasite that produces this disease; such as the life cycle, epidemiology, clinical manifestations, socioeconomic problem, as well as diagnostic techniques, infection mechanisms, treatment and control programs that have been has been carried out in various developed countries with the aim of eradicating the disease. Context: Cysticercosis human is a zoonotic disease which is produced by the accidental consumption of T. solium eggs; This can cause serious health problems and even death. While prevalence data are available throughout the world, the incidence rate and the cumulative incidence figures are insufficient, which limits the understanding of the T. solium epidemiology is considered as one of the infections that occurs most frequently in the system nervous; It is also endemic in several countries in Latin America, Asia and Africa. Methodology: This article was carried out a bibliographic review of the information available on cysticercosis and its causative parasite T. solium; as well as the control programs that were carried out in the developed countries and that managed to eradicate this disease.
\end{abstract}

Key words: Cysticercosis, Taenia solium, neurocysticercosis.

\section{Introducción}

Taenia solium es un cestodo que puede causar dos infecciones diferentes en humanos, teniasis intestinal (la infección intestinal con la tenia adulta) y cisticercosis (una infección tisular con larvas quísticas o cisticerco). ${ }^{1} \mathrm{La}$ cisticercosis humana $(\mathrm{CCH})$ es una enfermedad parasitaria causada por el desarrollo del estadío larvario del metacestodo de Taenia solium (cisticerco) en los músculos, el sistema nervioso central (causante de neurocisticercosis (NCC)), el tejido subcutáneo y los ojos (que causa cisticercosis subcutánea y ocular, respectivamente). ${ }^{2}$ En los humanos, la enfermedad es responsable de diferentes manifestaciones clínicas que pueden ser muy graves e incluso causar la muerte. $^{3}$

La cisticercosis/teniasis de Taenia solium es ampliamente endémica en la mayoría de los países en desarrollo, donde representa aproximadamente un tercio de todos los casos de convulsiones y epilepsia. ${ }^{4}$ La presencia del parásito está relacionada con condiciones sanitarias deficientes, higiene inadecuada, defecación al aire libre, presencia de cerdos en libertad y pobreza. ${ }^{5}$ El Grupo de Trabajo Internacional para la Erradicación de Enfermedades (International Task Force for Disease Eradication (ITFDE)) de la Organización 
Mundial de la Salud (OMS) declaró a Taenia solium como potencialmente erradicable en $1992{ }^{6}$

Los seres humanos se infectan por el consumo de carne de cerdo cruda o poco cocida infectada con cisticercos, lo que resulta en el desarrollo de una tenia intestinal adulta (teniasis). ${ }^{7}$ Las manifestaciones clínicas de la cisticercosis son bastante conocidas; sin embargo, la información acerca de su epidemiología es escasa. ${ }^{8}$ En un estudio que se desarrolló en México aproximadamente el $9 \%$ de las hospitalizaciones en los departamentos de neurología y neurocirugía fueron a causa de casos de neurocisticercosis, además de ser establecidos como diagnóstico final de alrededor del $11 \%$ al $25 \%$ de los pacientes que fueron operados para extirpar tumores del encéfalo. También se encontraron cisticercosis cerebrales en un porcentaje del $2,8 \%$ al 3,6\% de las necropsias realizadas en la ciudad de México y se presentaron informes que establecían que la cisticercosis fue la enfermedad causante de la muerte de los pacientes hospitalizados en un porcentaje del $0,6 \%$ al $1,5 \%$. ${ }^{9}$ Si bien el ciclo de vida de T. solium es conocido y existen diferentes enfoques para interrumpir la transmisión, se necesitan mejores esfuerzos para comprender los factores que afectan la dinámica de transmisión del parásito a fin de diseñar intervenciones adecuadas que puedan integrarse en los programas de control. ${ }^{10}$

\section{Taenia solium - Generalidades}

La infección por Taenia solium y la neurocisticercosis resultante de esta infección son endémicas en aquellos países que son menos desarrollados donde los cerdos son criados como fuente de alimento. ${ }^{11,12}$ La neurocisticercosis es común en América Latina, la mayor parte de Asia, el África y algunas partes de Oceanía, y constituye la principal causa de epilepsia adquirida en todo el mundo. ${ }^{13}$ En la actualidad es diagnosticada con mayor frecuencia en los países más desarrollados debido a la inmigración de portadores de solitaria de zonas endémicas. ${ }^{14}$

\subsection{Ciclo de Vida de T. solium}

Taenia solium tiene dos huéspedes, los seres humanos que son los huéspedes definitivos y los cerdos actúan como huéspedes intermediarios y albergan larvas o cisticercos. ${ }^{13}$ Después de que los seres humanos ingieren cisticercos viables, la tenia que en un comienzo es únicamente una larva crece en un gusano adulto dentro de alrededor dos meses en el intestino delgado humano. Los huevos o los segmentos de gusanos más distales (proglótidos) que contienen huevos maduros se liberan / desprenden del gusano intermitentemente y luego se pasan al medio ambiente con heces humanas. ${ }^{15}$ Los cerdos se infectan por la ingestión de huevos de T. solium que se encuentran en las heces humanas infectadas, a través del comportamiento coprófago o por la ingestión de agua o alimentos contaminados, y desarrollan cisticercosis porcina. ${ }^{7}$ En el intestino de los cerdos, los huevos eclosionan, liberando los embriones, que cruzan la mucosa intestinal para llegar al torrente sanguíneo y son transportados a diferentes tejidos. ${ }^{16}$

La neurocisticercosis (NCC) es la enfermedad parasitaria más importante del sistema nervioso central y la principal causa de epilepsia adquirida en áreas endémicas de $T$. solium, donde la NCC se asocia con $14,2 \%$ a $50 \%$ de los casos de epilepsia. ${ }^{17}$

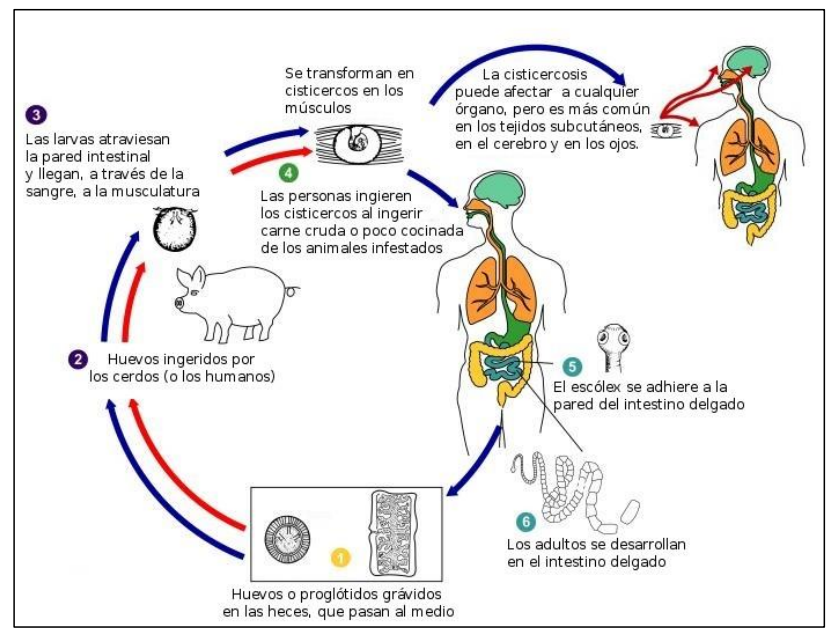

Fig. 1. Ciclo de Vida de Taenia solium

Recuperado de: http://animalandia.educa.madrid.org

\subsection{Epidemiología}

En América Latina, la infección ha sido reportada en al menos 18 países y se considera un importante problema de salud pública, en especial en las zonas rurales pobres. ${ }^{18} \mathrm{La}$ región andina de Ecuador y los países vecinos es hiperendémica para la cisticercosis. ${ }^{19}$ La neurocisticercosis es por lo general aceptada como la mayor causa de epilepsia adquirida en los países en desarrollo, y estudios controlados recientes con tomografía computarizada (TC) en Honduras, Ecuador y Perú han demostrado una asociación sólida entre neurocisticercosis y convulsiones en el campo, con cerca del $30 \%$ de las convulsiones que han sido atribuidas a la infección por neurocisticercosis (Medina et al., 2005). En el Ecuador, cerca del el $10 \%$ de todos los casos reportados de epilepsia, y el $25 \%$ de aquellos que se atribuyen a un hecho particular identificable, fueron a causa de cisticercosis del sistema nervioso central. ${ }^{20}$

La distribución de la cisticercosis porcina, de acuerdo con la OMS (1993), es muy alta en Bolivia, Brasil, Colombia, Ecuador, Guatemala, Honduras, México, Nicaragua, Perú y Venezuela, teniéndose como los más afectados, Perú del 7,1-26,9\% y Bolivia $22 \% .^{21}$ Estudios realizados en comunidades rurales endémicas ecuatorianas han demostrado una exposición al parásito que varía entre el 25 y el $40 \%$ y una proporción de individuos infectados que varía entre el 2,25 y el $4,99 \% .^{22}$

Ecuador tiene una población de alrededor de 16 millones. La población rural de Ecuador representa el $38 \%$ de la 
población total del país y está compuesta en su mayoría por las comunidades indígenas dedicadas a labores agrícolas. La región de la Sierra y la costa comprenden la mayoría de las zonas agrícolas activas. ${ }^{23}$

La cisticercosis es una de las 17 principales enfermedades tropicales desatendidas (ETD) identificadas por la OMS como un foco de investigación y control. ${ }^{15}$ En áreas donde $T$. solium es endémico, se sabe que la cisticercosis es la principal causa de convulsiones / epilepsia. ${ }^{24,25}$ Las revisiones sistemáticas indican que la convulsión / epilepsia fue la manifestación más común entre los pacientes diagnosticados de NCC (Carabin et al., 2011) y la proporción de casos de NCC entre las personas con epilepsia en países endémicos se estimó en $29.0 \% .^{17}$

Además de acuerdo con la OMS en el año 2013, el número de casos de cisticercosis humana estimado fue de 3-6 millones en China y 11-29 millones en la región de América Latina. ${ }^{15}$

En Ecuador la taza de diagnósticos de neurocisticersosis en los hospitales entre los años 1978 y 1984 se incrementaron de $0,3 \%$ a $2,62 \%$ por cada 1000 pacientes. ${ }^{26}$ En otro estudio realizado con anterioridad se estableció que la prevalencia de la neurocisticercosis en Cuenca-Ecuador fue de 1,3\% durante los años 1963-1981. ${ }^{27}$ Por otro lado, en varios exámenes que se realizaron en la materia fecal en poblaciones en 1984 la prevalencia de la teniasis humana en las muestras que se examinaron varió entre 0,03 y 3,23\%. Además en el Ecuador el $61 \%$ de los cerdos son criados al aire libre en granjas cuyo tamaño no alcanza ni a las diez hectáreas y adicionalmente en este mismo año solo el $48 \%$ de los cerdos sacrificados fueron sometidos a una revisión periódica con el veterinario. ${ }^{28}$

La NCC es endémico en América Central y del Sur, África Sub-sahariana y el sudeste de Asia, lo que constituye un desafío de salud pública para la mayoría del mundo en desarrollo. ${ }^{29}$ Existen casi 50 millones de personas infectadas en todo el mundo y 50,000 casos de muerte se reportan cada año como resultado. ${ }^{30}$ Recientemente, ha habido un aumento en la prevalencia de NCC en América del Norte debido al aumento de la inmigración de las regiones endémicas. ${ }^{31}$ Los Estados Unidos y Canadá, aunque no son endémicos, informan más de 5 casos por año. ${ }^{32}$

Informes oficiales del Ministerio de Salud Pública de Ecuador resaltan la información de 67 nuevos casos de cisticercosis en 2013 o 0,42 casos por cada 100.000 personas. La mayoría de estos casos corresponden a pacientes hospitalizados con síntomas de neurocisticercosis solo en hospitales públicos, lo que sugiere una gran subestimación. ${ }^{1}$ Los datos muestran una tendencia decreciente desde 1994 con 400 casos nuevos o 3,6 casos por cada 100.000 habitantes en ese momento. La región de la Sierra parece ser la principal responsable, y las provincias de Pichincha y Loja muestran el mayor número de casos y casos notificados por cada 100.000 personas. ${ }^{24}$ La tendencia decreciente a lo largo del tiempo es compatible con lo observado por especialistas en grandes hospitales en Ecuador y podría ser causada por la mejora de las condiciones generales de vida, como un mejor saneamiento y un mejor acceso a la atención médica; con las mejoras generales en el sistema de salud del Ecuador, es posible que los pacientes que presentan síntomas sean diagnosticados y tratados de forma adecuada en una etapa temprana de la enfermedad evitando la progresión de la enfermedad y reduciendo el número de casos hospitalizados, mientras que el número de pacientes asintomáticos o los casos no hospitalizados se mantienen estables. $^{33}$

\subsection{Importancia Clínica}

La cisticercosis humana puede involucrar el tejido muscular esquelético, el miocardio, el ojo y el tejido subcutáneo, ${ }^{34}$ sin embargo, este tipo de manifestaciones se consideran menos comunes o patógenos en comparación con neurocisticercosis (NCC). ${ }^{35}$ Los trastornos clínicos más frecuentes descritos para NCC incluyen epilepsia y convulsiones, seguidos por dolores de cabeza, déficits neurológicos focales y trastornos relacionados con el aumento de la presión intracraneal como hidrocefalia o papiledema. Otras manifestaciones no tan frecuentes son: meningitis, trastornos visuales, trastornos cognitivos y otras manifestaciones psiquiátricas relacionadas con estados mentales alterados. $^{36}$

En la NCC los mecanismos patogénicos varían según la ubicación de los parásitos, su volumen y la respuesta inflamatoria del paciente, que a su vez se relaciona con la etapa evolutiva de los parásitos y su ubicación dentro o fuera del parénquima nervioso.

En la cisticercosis humana sintomática los quistes parásitos se establecen, sobreviven durante un período extremadamente variable que puede ir de meses a muchos años, eventualmente se resuelven siguiendo un proceso involutivo para luego desaparecer y por lo general reaparecen más tarde como una cicatriz calcificada (ESCOBAR, 1983). El NCC intraparenquimatoso se asocia principalmente con convulsiones y cefalea, y la opinión general es que evolucionará hacia la resolución, aunque este proceso puede llevar al paciente durante muchos años con convulsiones con los riesgos, costos y daños posteriores a la calidad de vida del paciente. ${ }^{37}$

De acuerdo con Carabin et al., 2017 se encuentran disponibles un número creciente de estudios clínicos y epidemiológicos sobre NCC y cisticercosis ha resultado en la publicación de varias revisiones sistemáticas, las cuales han confirmado creencias arraigadas de que alrededor del $30 \%$ de las personas que viven con epilepsia en países endémicos para la cisticercosis muestran lesiones NCC en el cerebro (Fig. 2). ${ }^{38}$

\subsection{Diagnóstico}

La teniasis puede ser diagnostocada principalmente con un examen coproparasitario (técnica de sedimentación, flotación y frotis grueso), también existe la técnica de raspado perianal; hay que tener en consideración que estos 
exámenes no siempre pueden distinguir entre T. solium y T. saginata por ello no poseen la confiabilidad deseada. ${ }^{39}$

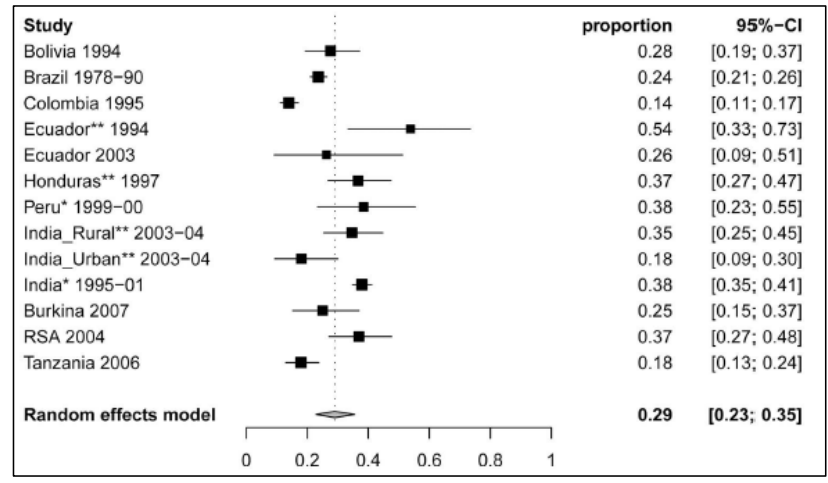

Fig. 2. Diagrama de bosque de la proporción de NCC (IC del 95\%) en personas con epilepsia a partir de 12 estudios que informan de casos en todos los grupos de edad. * Indica estudios entre personas con epilepsia y ataques. ** Indica estudios solo entre personas con epilepsia activa. La figura, el título y la leyenda fueron tomados de la Figura 4 en (Ndimubanzi et al., 2010) (http://dx.doi.org/10.1371/journal.pntd.0000870.g004). No se realizaron cambios en la figura original, el título y la leyenda en (Ndimubanzi et al., 2010)

Por otro lado, la neurocisticercosis es diagnosticada por medio de exámenes de imagen (tomografía computarizada o resonancia magnética) (Figura 3 y 4), y luego confirmados por serología con la técnica de western blot. ${ }^{40}$

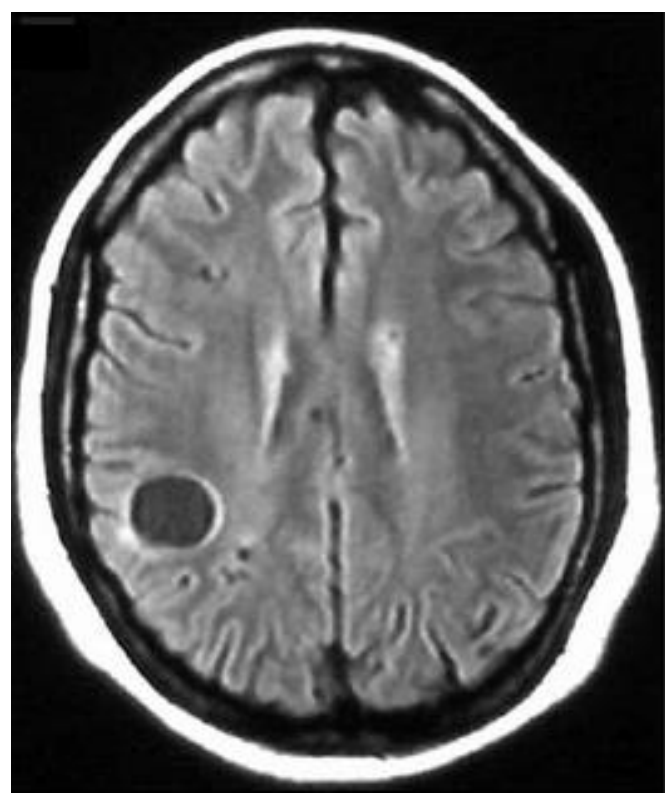

Fig. 3. Resonancia magnética cerebral Recuperado de:

http://www.scielo.org.pe/img/revistas/rins/v27n4/a16fig01a.jpg

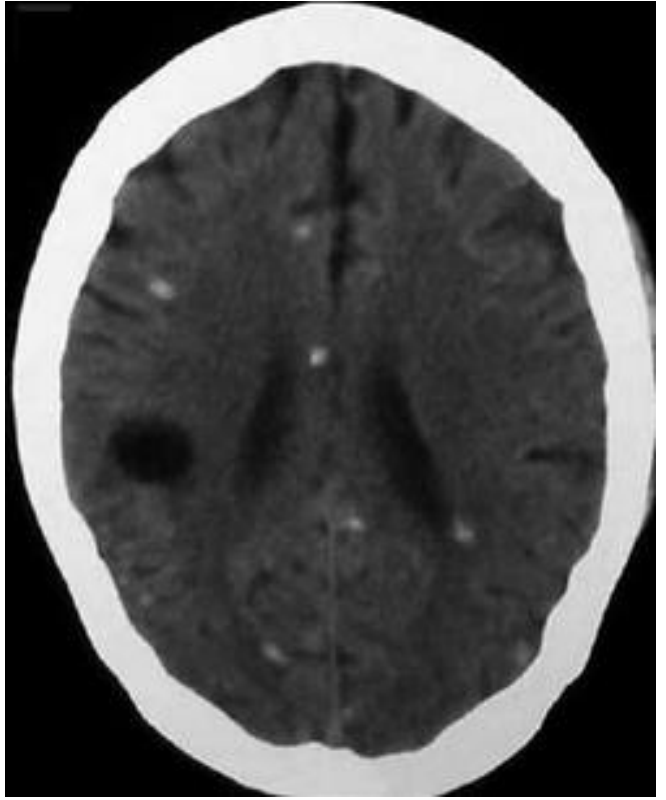

Fig. 4. Tomografía computarizada cerebral Recuperado de:

http://www.scielo.org.pe/img/revistas/rins/v27n4/a16fig01a.jpg

\subsection{Mecanismos de infección}

Dentro de los mecanismos habituales de infección por cisticercosis están la heteroinfección, que está relacionada íntimamente con las condiciones sanitarias generales y la autoinfección que se refiere a las condiciones higiénicas individuales. ${ }^{39}$ A pesar de lo mencionado se han realizado algunos estudios que plantean que la cisticercosis se distribuye dentro de la población de tal forma que no se relaciona con factores socio-económicos, ambientales ni tampoco higiénicos. ${ }^{41}$

Existen algunos factores que determinan la transmisión de la cisticercosis:

- Realizar deposiciones al ras del suelo, provocando contaminación de alimentos, agua y aire, debido a la presencia de moscas.

- Riego de cultivos de verduras y frutas con aguas negras, provenientes de las zonas urbanas.

- Educación higiénica sanitaria deficiente dentro de la población.

\subsection{Tratamiento}

Se ha experimentado algunos medicamentos para el tratamiento de la teniasis, el que se ha dado resultados muy buenos es la niclosamida que es utilizada en dosis únicas orales de $2 \mathrm{~g}$ en adultos y $1 \mathrm{~g}$ en niños, también puede emplearse el praziquantel en dosis de 5 a $10 \mathrm{~g} / \mathrm{kg} ;{ }^{242}$ Para tratar la neurocisticercosis, se debe realizar un tratamiento sintomático el que tiene la finalidad de ayudar en el control de las manifestaciones clínicas principalmente las crisis epilépticas; y el tratamiento antiparasitario va a depender de estado, número, tamaño y localización de los quistes, y generalmente se utiliza albendazol en una dosis de 15 
$\mathrm{mg} / \mathrm{kg} / \mathrm{d}$ por una a dos semanas o praziquantel con una dosis de $50 \mathrm{mg} / \mathrm{kg} / \mathrm{d}$ por dos semanas, usualmente con esteroides. $^{35}$

\subsection{Salud Pública e importancia Socio-económica}

T. solium no es únicamente la causante de grave enfermedad zoonótica, sino que también provoca grandes pérdidas económicas en la industria porcina y a nivel hospitalario ${ }^{43}$

De acuerdo con Román et al (2000) se reportaron 50000 muertes por año al intentar hacer una estimación global de las muertes causadas por cisticercosis humana $(\mathrm{CCH}),{ }^{44}$ pero de acuerdo a estudios más recientes, se presentaron estimaciones de la Carga Global de la Enfermedad (GBD) para 2010 y 2013 las cuales reportaron entre 1200 y 700 muertes anuales, respectivamente, atribuidas a $\mathrm{CCH} .{ }^{45}$

\subsection{Programas de control}

Las estrategias propuestas para controlar las infecciones por $T$. solium se basan en la interrupción del ciclo de vida del parásito, algunas de ellas adaptadas de las experiencias aprendidas en los países desarrollados donde la enfermedad fue erradicada de manera eficiente. ${ }^{1}$ A continuación, se detallan las estrategias disponibles para la teniasis de $T$. solium de acuerdo con con Carabin et al. (2014) ${ }^{10}$; Singh \& Prabhakar $(2002)^{18}$ y actualizado por Thys et al. $(2015)^{46}$ ; Braae et al.(2015) ${ }^{47}$; Gilman et al.(2012) ${ }^{48}$; Bulaya et al. $(2015)^{49}$; Miwunda et al. $(2015)^{50}$.

Eliminación de cadáveres de cerdos infectados (inspección de carne): actividad de bajo costo, pero también de baja sensibilidad.

Congelar la carne: la congelación de carne puede matar los quistes parásitos, se requieren largos períodos de tiempo para matar los quistes en la carne congelada.

Mejora de las prácticas de cocción: el tratamiento térmico adecuado de la carne puede matar los quistes, no consumir carne cruda o a medio coser.

Educación para la salud: nuevos medios ahora ampliamente disponibles, requieren seguimiento a largo plazo.

Tratamiento de la teniasis intestinal (intervenciones dirigidas): elimina el riesgo de transmisión significativo conocido y requeriría intervenciones repetidas para el control a largo plazo.

Tratamiento de la teniasis intestinal (administración masiva de medicamentos): costo logístico reducido en comparación con otras estrategias; sin embargo, la transmisión se recupera rápidamente después de suspender el tratamiento.

Mejora de la cría de cerdos: proporciona beneficios más allá del control de T. solium, pero es demasiado costoso para los agricultores pobres.

Vacunación de cerdos: protección a largo plazo, no accesible para agricultores pobres que ni siquiera tienen dinero para alimentar a sus cerdos.

Quimioterapia de cerdos infectados: altamente eficaz, se necesitan de 3 meses para la desaparición completa de los quistes cuando se usa oxfendazol.

\section{Conclusiones}

La cisticercosis es una enfermedad que se la considera endémica principalmente en países de América Central y del Sur, África Sub-sahariana y el sudeste de Asia, sin embargo, a causa de las migraciones de personas de estos países hacia otros lugares, esta enfermedad se ha presentado en países en donde no es endémica.

A pesar de que constituye una preocupación para los países en donde la enfermedad es endémica, debido a que existen diferentes programas de control que han servido para romper el ciclo de vida de $T$. solium, al menos en algunos países desarrollados han logrado erradicar esta enfermedad; sin embargo, en los países de Latinoamérica entre los que se incluye el nuestro, existe bastante pobreza y las estructuras sanitarias son inadecuadas y deficientes, por lo que esta enfermedad todavía prevalece.

En Ecuador, Perú, México entre otros países, se han realizado varios estudios de la cisticercosis y las consecuencias que esta genera para las personas que la padecen, principalmente para aquellos pacientes que padecen de neurocisticercosis que es la que produce manifestaciones clínicas más graves por ejemplo la epilepsia; en estos países se considera la cisticercosis como una enfermedad de declaración obligatoria.

\section{Referencias Bibliográficas}

1. Coral M. Epidemiological transmission patterns of Taenia solium cysticercosis in endemic areas : the case of Ecuador; 2016.

2. Carpio A, Escobar A, Hauser WA. Cysticercosis and Epilepsy: A Critical Review. Epilepsia. 2005 aug;39(10):1025-1040.

3. Sorvillo FJ, DeGiorgio C, Waterman SH. Deaths from Cysticercosis, United States. Emerging Infectious Diseases. $2007 \mathrm{feb}$;13(2):230-235.

4. Neurocysticercosis: updated concepts about an old disease. The Lancet Neurology. 2005 oct;4(10):653-661.

5. Lustigman S, Prichard RK, Gazzinelli A, Grant WN, Boatin BA, McCarthy JS, et al. A Research Agenda for Helminth Diseases of Humans: The Problem of Helminthiases. PLOS Neglected Tropical Diseases. 2012 apr;6(4):e1582.

6. and C. Recommendations of the International Task Force for Disease Eradication. Morb Mortal Wkly Rep. 1993;42(16):1-38.

7. Coral M, Rodríguez R, Celi M, García HH, Rodríguez $\mathrm{S}$, Devleesschauwer B, et al. Incidence of Human Taenia solium Larval Infections in an Ecuadorian Endemic Area: Implications for Disease Burden Assessment and Control. PLOS Neglected Tropical Diseases. 2014 may;8(5):e2887.

8. Cruz M, Davis A, Dixon H, Pawlowski Z, Proano J. Estudios operativos sobre el control de la teniasis/cisticercosis por Taenia solium en el Ecuador. Bol Of Sanit Panam. 1990;108(2):113-122. 
9. Sarti EJ, Schantz PM, Lara R, Gomez HD, Flisser A. 24. Del Brutto OH, Del Brutto VJ. Changing pattern of Taenia solium taeniasis and cysticercosis in a Mexican village. Tropical medicine and parasitology : official organ of Deutsche Tropenmedizinische Gesellschaft and neurocysticercosis in an urban endemic center (Guayaquil, Ecuador). Journal of the Neurological Sciences. 2012 apr;315(1):64-66.

of Deutsche Gesellschaft fur Technische Zusammenarbeit 25. Moyano LM, Saito M, Montano SM, Gonzalvez G, Olaya (GTZ). 1988 sep;39(3):194-198.

10. Carabin H, Traoré AA. Taeniasis and Cysticercosis Control and Elimination Through Community-Based Interventions. Current Tropical Medicine Reports. 2014 dec;1(4):181-193.

$\mathrm{S}$, Ayvar V, et al. Neurocysticercosis as a Cause of Epilepsy and Seizures in Two Community-Based Studies in a Cysticercosis-Endemic Region in Peru. PLOS Neglected Tropical Diseases. 2014 feb;8(2):e2692.

11. Garcia HH, Brutto OH. TAENIA SOLIUM CYSTICERCOSIS. Infectious Disease Clinics. 2000 mar;14(1):97119.

26. Emzo F, álvarez J. Prevalencia y seguimiento epidemiológico de la teniasis y cisticercosis. Rev Cienc Vet. 1985;5:51-81.

12. White AC. Neurocysticercosis: Updates on Epidemiology, Pathogenesis, Diagnosis, and Management. Annual Re-28. Ruiz P. Teniasis cisticercosis en el Ecuador. Rev Divulg view of Medicine. 2000;51(1):187-206.

27. Cordero L, Ugalde J. Cisticercosis. 29-32,1984. Trib Med Quito. 1984;p. 29-32.

Cient Ecuador. 1986;1:85-98.

13. García HH, Gonzalez AE, Evans CA, Gilman RH. 29. Yacoub HA, Goldstein I, El-Ghanem M, Sharer L, SouaTaenia solium cysticercosis. The Lancet. 2003 aug;362(9383):547-556.

yah N. Spinal racemose cysticercosis: case report and review. Hospital Practice. 2017 may;45(3):99-103.

14. Schantz PM, Wilkins PP, Tsang VCW. Immigrants, Ima- 30. Mahale RR, Mehta A, Rangasetty S. Extraparenchymal ging, and Immunoblots: the Emergence of Neurocysticercosis as a Significant Public Health Problem. Emerging Infections 2. 1998 jan;p. 213-242.

(Racemose) Neurocysticercosis and Its Multitude Manifestations: A Comprehensive Review. Journal of Clinical Neurology. 2015 jul;11(3):203-211.

15. Wu HW, Ito A, Ai L, Zhou XN, Acosta LP, Lee Willing- 31. Del la Garza Y, Graviss EA, Daver NG, Gambarin KJ, ham III A. Cysticercosis/taeniasis endemicity in Southeast Asia: Current status and control measures. Acta Tropica. 2017 jan;165:121-132.

16. Zoli A, Shey O, Assana E, Nguekam JP, Dorny P, Brandt J, et al. Regional status, epidemiology and impact of Taenia 32. World Health Organization. Endemicity of Taenia solium; solium cysticercosis in Western and Central Africa. Acta Tropica. 2003 jun;87(1):35-42.

17. Ndimubanzi PC, Carabin H, Budke CM, Nguyen H, Qian YJ, Rainwater E, et al. A Systematic Review of the Frequency of Neurocyticercosis with a Focus on People Shandera WX, Schantz PM, et al. EPIDEMIOLOGY OF NEUROCYSTICERCOSIS IN HOUSTON, TEXAS. The American Journal of Tropical Medicine and Hygiene. 2005 oct;73(4):766-770. 2015.

33. Alarcón TA, Del Brutto O. Neurocysticercosis: declining incidence among patients admitted to a large public hospital in Guayaquil, Ecuador. Pathogens and Global Health. 2012 sep;106(5):310-311.

with Epilepsy. PLOS Neglected Tropical Diseases. 201034. Takayanagui OM, Chimelli L. Disseminated muscular feb;4(11):e870.

18. Singh G, Prabhakar S. Taenia Solium Cysticercosis: From Basic to Clinical Science. CABI; 2002. cysticercosis with myositis induced by praziquantel therapy. The American Journal of Tropical Medicine and Hygiene. 1998 dec;59(6):1002-1003.

19. Cruz M, Davis A, Dixon H, Pawlowski ZS, Proano J.35. Garcia HH, Gonzalez AE, Rodriguez S, Gonzalvez G, Operational studies on the control of Taenia solium taeniasis/cysticercosis in Ecuador. Bulletin of the World Health Organization. 1989;67(4):401-407.

20. Organización Mundial de la Salud. Control de la neurocisticercosis. 56 a ASAMBLEA MUNDIAL DE LA SALUD; 36. Carabin H, Ndimubanzi PC, Budke CM, Nguyen H, Qian 2003.

21. Zhunio M. índice de prevalencia de cisticercosis en cerdos faenados en el camal municipal del cantón Pasaje provincia de El Oro. Machala; 2015.

22. Rodriguez R, Benitez W, Praet N, Saa LR, Vercruysse $\mathrm{J}$, Brandt $\mathrm{J}$, et al. Taeniasis-cysticercosis in Southern Ecuador: assessment of infection status using multiple la- 38 boratory diagnostic tools. Memórias do Instituto Oswaldo Cruz. 2006 nov;101(7):779-782.

23. Instituto Nacional de Estadísticas y Censos (INEC). III Llanos-Zavalaga F, Tsang VCW, et al. Epidemiología y control de la cisticercosis en el Perú. Revista Peruana de Medicina Experimental y Salud Pública. 2010 dec;27:592-597.

Y, Cowan LD, et al. Clinical Manifestations Associated with Neurocysticercosis: A Systematic Review. PLOS Neglected Tropical Diseases. 2011 may;5(5):e1152.

37. Gonzales I, Rivera J, García H. Pathogenesis of Taenia solium taeniasis and cysticercosis. Parasite Immunology. 2016 jan;38(3):136-146.

8. Carabin H, Winkler AS, Dorny P. Taenia solium cysticercosis and taeniosis: Achievements from the past 10 years and the way forward. PLOS Neglected Tropical Diseases. 2017 apr;11(4):e0005478.

Censo Nacional Agropecuario. 1 ed. Quito Ecuador: Mi-39. Sartí EJ. La taeniasis y cisticercosis en México (renisterio de Agricultura.; 2002. visión bibliográfica). Salud Pública de México. 2014 
nov;28(5):556-563.

40. Del Brutto OH, Rajshekhar V, White AC, Tsang VC, Nash 48 TE, Takayanagui OM, et al. Proposed diagnostic criteria for neurocysticercosis. Neurology. 2001 jul;57(2):177183.

41. Flisser A. Taeniasis and cysticercosis due to Taenia 49. solium. Progress in Clinical Parasitology. 1994;4:77-116.

42. Pearson RD, Hewlett EL. Niclosamide therapy for tapeworm infections. Annals of Internal Medicine. 1985 apr;102(4):550-551.

43. González T, Barboza M. Neurocisticercosis en niños. 50. Mwidunda SA, Carabin H, Matuja WBM, Winkler AS, Revista Ciencias Biomédicas. 2011;2(2).

44. Román G, Sotelo J, Del Brutto O, Flisser A, Dumas M, Wadia $\mathrm{N}$, et al. A proposal to declare neurocysticercosis an international reportable disease. Bulletin of the World Health Organization. 2000 mar;78:399-406.

45. Lozano R, Naghavi M, Foreman K, Lim S, Shibuya K, Aboyans V, et al. Global and regional mortality from 235 causes of death for 20 age groups in 1990 and 2010: a systematic analysis for the Global Burden of Disease Study 2010. The Lancet. 2012 dec;380(9859):2095-2128.

46. Thys S, Mwape KE, Lefèvre P, Dorny P, Marcotty T, Phiri AM, et al. Why Latrines Are Not Used: Communities' Perceptions and Practices Regarding Latrines in a Taenia solium Endemic Rural Area in Eastern Zambia. PLOS Neglected Tropical Diseases. 2015 apr;9(3):e0003570.

47. Braae UC, Harrison W, Lekule F, Magnussen P, Johansen MV. Feedstuff and poor latrines may put pigs at risk of cysticercosis - A case-control study. Veterinary Parasi- tology. 2015 nov;214(1):187-191.

Gilman RH, Gonzalez AE, Llanos F, Tsang VCW, Garcia HH. Prevention and control of Taenia solium taeniasis/cysticercosis in Peru. Pathogens and Global Health. 2012 sep;106(5):312-318.

C, Mwape KE, Michelo C, Sikasunge CS, kungu C, Gabriel S, et al. Preliminary evaluation of Community-Led Total Sanitation for the control of Taenia solium cysticercosis in Katete District of Zambia. Veterinary Parasitology. 2015 jan;207(3):241-248.

Ngowi HA. A School Based Cluster Randomised Health Education Intervention Trial for Improving Knowledge and Attitudes Related to Taenia solium Cysticercosis and Taeniasis in Mbulu District, Northern Tanzania. PLOS ONE. 2015 feb;10(2):e0118541.

Recibido: 1 de mayo de 2018

Aceptado: 13 de septiembre de 2018

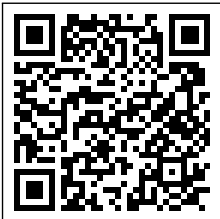


